\title{
Copying holograms using light of reduced spatial and temporal coherence
}

\author{
I. Pascual, A. Beléndez, F. Mateos, and A. Fimia
}

\begin{abstract}
A reduced spatial and temporal coherence light from a halogen lamp is used to copy holographic optical elements, and a maximum diffraction efficiency of $80 \%$ is achieved.

Key words: Holography, hologram copying, reduced coherence.
\end{abstract}

The obtention of holographic optical elements (HOE's) that can be recorded by use of a direct method with coherent light sources is limited because the radiation emitted by lasers currently available does not correspond to the regions of high spectral sensitivity of suitable recording materials. One technique for solving this problem is the recording of the HOE's with a copying method that uses reduced spatial and temporal coherence light. ${ }^{1}$

The reduced coherence light is from a highpressure mercury lamp or from a continuous halogen lamp, which permits us to demonstrate the feasibility of our optical system. The idea is to substitute a discrete source, the high-pressure mercury lamp, with a continuous source, the halogen lamp. By making this change, we can evaluate the versatility of the setup because the light from this continuous lamp is still partially coherent but to a lesser degree. However, for the angular size of the source and therefore for the specific bandwidth it does provide enough coherence to copy HOE's.

Experiments were carried out with dichromated gelatin (DCG) from Kodak $649 \mathrm{~F}$ plates. In order to obtain holographic gratings in DCG by use of reduced spatial and temporal coherence light (halogen lamp), we used a two-step method for recording HOE's with partially coherent light. ${ }^{1}$

The method consists of the copying of a previously achieved holographic grating in the Fresnel's region so that, once it is placed at Bragg's angle, and once its

I. Pascual, F. Mateos, and A. Fimia are with the Laboratorio de Optica, Departamento Interuniversitario de Optica, Universidad de Alicante, Apartado 99, Alicante E-03080, Spain; A. Beléndez is with the Departamento de Ingeniería de Sistemas y Comunicaciones.

Received 10 December 1992

0003-6935/93/326456-02\$06.00/0.

(1) 1993 Optical Society of America. object and reference beams in reconstruction are optimized to a 1:1 ratio in the second stage, it is possible to copy a second holographic grating in a DCG, the diffraction efficiency of which is comparable with the one obtained by use of a high-pressure mercury lamp. The basic experimental setup is shown in Fig. 1. This system is not described here in any detail since it is essentially the same as that used by Pascual et al., ${ }^{1}$ but here we substitute the mercury lamp with a halogen lamp. A collimated beam from a $240-\mathrm{W}$ continuous halogen lamp was used to make the copy. The angular size of the source was 0.015 rad (9 $\mathrm{mm}$ in diameter) and the bandwidth was 20 $\mathrm{nm}$, which would make the spatial and temporal coherence factor ${ }^{2}$ over 0.8 if the holographic gratings have a spatial frequency of 1000 lines $/ \mathrm{mm}$ and as long the space between the master and the copy is less than $10 \mu \mathrm{m}$. When the spatial frequency of the holographic gratings increases to 2000 lines $/ \mathrm{mm}$, the spatial and temporal coherence factor decreases to 0.6 .

A series of transmission gratings with exposures in the range $1-100 \mathrm{~mJ} / \mathrm{cm}^{2}$ was copied. The spatial frequency stored was 1000 lines $/ \mathrm{mm}$. The variation

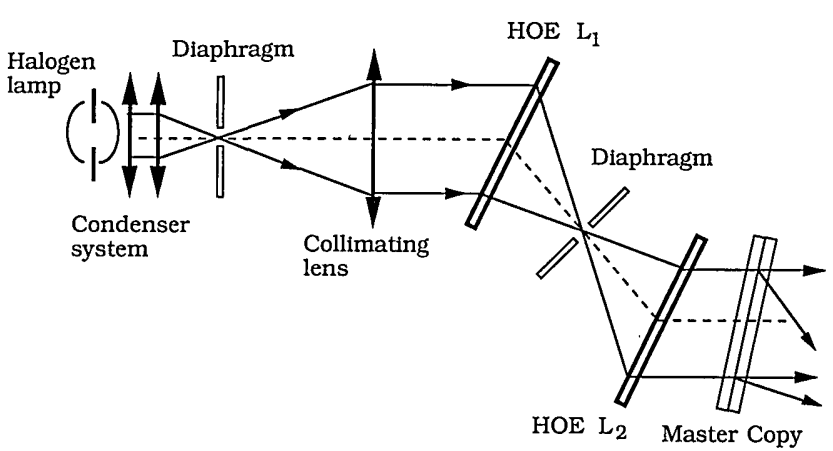

Fig. 1. Experimental setup: $\mathrm{HOE} \mathrm{L}_{1}, \mathrm{HOE} \mathrm{L}_{2}$, holographic optical element lenses. 


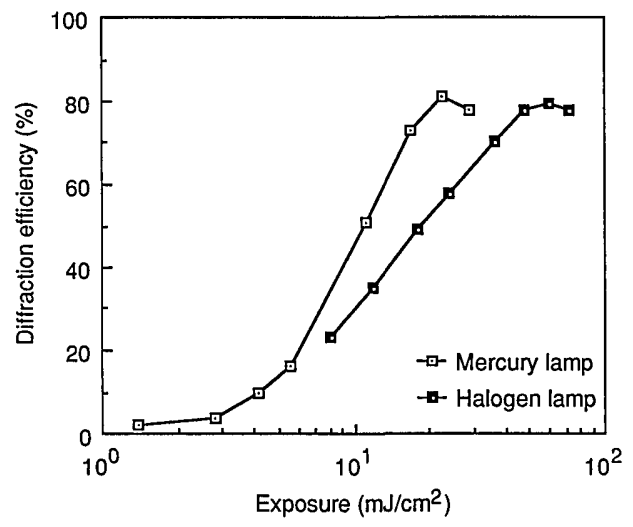

Fig. 2. Diffraction efficiency as a function of exposure for two sources: (a) high-pressure mercury lamp, (b) continuous halogen lamp.

in sensitivity ${ }^{3}$ in DCG is in the range 1-100 when we tune from 514 to $405 \mathrm{~nm}$; therefore we chose $405 \mathrm{~nm}$ as the recording wavelength.

After exposure, the plates were developed in a DCG standard developing process, and it was possible to compare the results obtained by this method with the results reported in the literature, ${ }^{4}$ obtained by use of the same recording material and process as those that we used when working with coherent sources.

The results are summarized in Fig. 2, which shows the diffraction efficiency versus exposure for two sources of irradiation, a high-pressure mercury lamp and a continuous halogen lamp. The measurement wavelength was $633 \mathrm{~nm}$. As can be seen in Fig. 2, the maximum diffraction efficiency achieved is the same with both types of lamps, $80 \%$. We also made holographic lenses with spatial frequencies that were in the range 600-2000 lines $/ \mathrm{mm}$ and that achieved the same diffraction efficiency and optical quality as in the gratings. We should point out that, for these lenses to be obtained, the spatial coherence factor was in the range $0.9-0.6$ on the same lens.

In conclusion, we can say that the results are satisfactory, as it is possible to obtain diffraction gratings and high-efficiency holographic lenses experimentally with reduced coherence light from a continuous source (a halogen lamp) even though exposure times are high, given that the usable intensity is lower. We can make an interesting observation on the stability of the system: if diffraction efficiencies of $\sim 80 \%$ are to be achieved, 3-hr exposure times are needed. ${ }^{5}$ Nevertheless, both the optical quality of the grating and its efficiency are comparable with those obtained with a mercury lamp, and therefore it is possible to choose the lamp that best fits within the experimental setup used with consideration of the specific application it is designed for.

\section{References}

1. I. Pascual, A. Beléndez, and A. Fimia, "Holographic system for copying holograms by using partially coherent light," Appl. Opt. 31, 3312-3319 (1992).

2. H. H. Hopkins, "The theory of coherence and its applications," in Advanced Optical Techniques, A. C. S. Van Heel, ed. (NorthHolland, Amsterdam, 1967), pp. 491-513.

3. L. Solymar and D. J. Cooke, Volume Holography and Volume Gratings (Academic, London, 1981), pp. 278-286.

4. D. Meyerhofer, "Dichromated gelatin," in Holographic Recording Materials, H. M. Smith, ed., Vol. 20 of Topics in Applied Physics (Springer-Verlag, Berlin, 1977), pp. 75-99.

5. I. Pascual, A. Beléndez, and A. Fimia, "Analysis of the holographic reciprocity law for dichromated gelatin," Appl. Opt. 31, 3200-3201 (1992). 